\title{
ON A SINGULAR QUASILINEAR ANISOTROPIC ELLIPTIC BOUNDARY VALUE PROBLEM
}

\author{
Y. S. CHOI, A. C. LAZER, AND P. J. McKENNA
}

\begin{abstract}
We consider the problem

$$
u^{a} u_{x x}+u^{b} u_{y y}+p(\mathbf{x})=0
$$

with $a \geq 0, b \geq 0$, on a smooth convex bounded region in $\mathbf{R}^{2}$ with Dirichlet boundary conditions. We show that if the positive function $p$ is uniformly bounded away from zero, then the problem has a classical solution.
\end{abstract}

\section{INTRODUCTION}

In [1], an anisotropic singular elliptic boundary value problem arises naturally when studying self-similar solutions of hyperbolic conservation laws in two dimensions. A comparison with the existing mathematical literature on singular elliptic boundary value problems revealed a curious gap: namely, there is a considerable amount of knowledge on the singular semilinear elliptic boundary value problem

$$
\begin{aligned}
u(\mathbf{x})^{\gamma} \Delta u+p(\mathbf{x}) & =0, \\
\left.u\right|_{\partial \Omega} & =0,
\end{aligned}
$$

where $\Omega$ is a sufficiently regular bounded domain in $\mathbf{R}^{N}, N \geq 1$, and $p$ is a sufficiently regular function which is positive on $\bar{\Omega}$. In the case $N=1$, this problem arises in certain applications in fluid mechanics and pseudoplastic flow (see $[5,6,7])$. The $N$-dimensional problem $(1.1)-(1.2)$ has been studied in [2] for general regions.

In [2], it is shown that solutions exist if $\Omega$ is $C^{3}$, and estimates are given for the behaviour of the solution as the boundary of $\Omega$ is approached. In particular, if $\gamma>1$, it is shown that solutions fail to be in $C^{1}(\bar{\Omega})$.

In [4], it was shown that if $\Omega$ has a somewhat less regular boundary, $p$ is positive on $\bar{\Omega}$ and $\gamma$ is any positive number, there is a unified simple proof that there is a unique solution of $(1.1)-(1.2)$, positive on $\Omega$, which is in $C^{2+\alpha}(\Omega) \cap$ $C(\bar{\Omega})$.

Received by the editors December 2, 1993 and, in revised form, April 13, 1994; originally communicated to the Proceedings of the AMS by Barbara L. Keyfitz.

1991 Mathematics Subject Classification. Primary 35J25.

Key words and phrases. Subsolution, supersolution, singular.

Research of the first author was partially supported by NSF grant DMS-9208636, of the second author by NSF grant DMS-9102233, and of the third author by NSF grant DMS-9102632. 
However, a small perturbation of the above problem, which is related to an equation arising in fluid dynamics, changes the situation totally, revealing problems which appear quite difficult, and on which we present some partial results in this paper.

None of the above-mentioned results apply in an obvious way to the problem

$$
\begin{aligned}
u^{a} u_{x x}+u^{b} u_{y y}+p(\mathbf{x}) & =0, \\
\left.u\right|_{\partial \Omega} & =0,
\end{aligned}
$$

where $a$ and $b$ are nonnegative, but not equal. (Without loss of generality, we let $a>b \geq 0$.)

The main problem appears to be that in the case $a=b$ monotonicity methods can be used, which do not adapt to the more general case.

In this paper, we shall present results on this problem when the region $\Omega$ is convex. We have difficulty believing that this restriction is essential, but have been unable to avoid it.

Our main theorem is:

Theorem 1. Let $\Omega \subset \mathbf{R}^{2}$ be a bounded convex domain with smooth boundary $\partial \Omega$ (of class $C^{2+\alpha}, 0<\alpha<1$ ). If $p \in C^{\alpha}(\overline{\mathbf{\Omega}}), p(\mathbf{x})>0$ for all $\mathbf{x} \in \bar{\Omega}$ and $\gamma>0$, then there exists a function $u \in C^{2+\alpha}(\Omega) \cap C(\bar{\Omega})$ such that $u(\mathbf{x})>0$ for all $\mathbf{x} \in \Omega$ and $u$ is a solution of (1.3)-(1.4).

Thus, in the next section, we shall describe a new comparison principle for quasilinear elliptic problems, and in the following section, we shall apply this principle to show the existence of solutions to problem (1.3)-(1.4). Finally, in the concluding section, we shall point out the many unsolved problems remaining in this area.

After submitting this paper, we found out that related results on the existence of weak solutions of a problem in divergence form were established independently in [1].

\section{A COMPARISON LEMMA}

In this section, using the maximum principle we will establish a comparison lemma on a class of quasilinear elliptic equations. This allows us to obtain a priori bounds on the solutions to this class of equations using the method of upper and lower solutions. Such bounds in turn give the existence of a solution to the equations (1.3)-(1.4) by a compactness argument.

Lemma 1. Given the equation

$$
f(u, \mathbf{x}) u_{x x}+u_{y y}+p(\mathbf{x}) g(u, \mathbf{x})=0,
$$

subject to the boundary condition

$$
\left.u\right|_{\partial \Omega}=\left.u_{0}\right|_{\partial \Omega}
$$

with $\left.u_{0}\right|_{\partial \Omega} \geq 0$, let $f, g$, and $p$ satisfy the conditions (A1)-(A3) respectively, where

(A1) $f:[0, \infty) \times \Omega \rightarrow[0, \infty)$ is continuous, and $f(\cdot, \mathbf{x})$ is nondecreasing for each $\mathbf{x} \in \Omega$.

(A2) $g:(0, \infty) \times \Omega \rightarrow(0, \infty)$ is continuous, and $g(\cdot, \mathbf{x})$ is nonincreasing for each $\mathbf{x} \in \Omega$. 
(A3) $p: \bar{\Omega} \rightarrow \mathbf{R}$ is continuous, and there exist positive constants $m$ and $M$ such that $0<m \leq p(\mathbf{x}) \leq M$ for all $\mathbf{x} \in \bar{\Omega}$.

Further we assume that

(L) there exists a lower solution $\varphi \in C(\bar{\Omega}) \cup C^{2}(\Omega)$ with $\varphi>0$ on $\Omega$ satisfying

$$
\begin{gathered}
f(\varphi, \mathbf{x}) \varphi_{x x}+\varphi_{y y}+p(\mathbf{x}) g(\varphi, \mathbf{x})>0, \\
\varphi_{x x} \leq 0
\end{gathered}
$$

on $\Omega$, and $\left.\varphi\right|_{\partial \Omega} \leq\left. u_{0}\right|_{\partial \Omega}$ [If $\varphi \geq \delta>0$ on $\bar{\Omega}$ for some $\delta>0$, then we can allow $\geq$ to be employed in equation (2.3).] and

(U) there exists an upper solution $\psi \in C(\bar{\Omega}) \cup C^{2}(\Omega)$ with $\psi>0$ on $\Omega$ satisfying

$$
\begin{gathered}
f(\psi, \mathbf{x}) \psi_{x x}+\psi_{y y}+p(\mathbf{x}) g(\psi, \mathbf{x}) \leq 0, \\
\psi_{x x} \leq 0
\end{gathered}
$$

on $\Omega$, and $\left.\psi\right|_{\partial \Omega} \geq\left. u_{0}\right|_{\partial \Omega}$.

If $u \in C(\bar{\Omega}) \cup C^{2}(\Omega)$ is any solution of equations (2.1) and (2.2) with $u>0$ on $\Omega$, then

$$
\varphi \leq u \leq \psi
$$

on $\bar{\Omega}$.

Remark 1. It is clear from the proof that assumptions (A1) and (A2) can be replaced by, respectively,

$\left(\mathbf{A} 1^{\prime}\right) \quad f: \mathbf{R} \times \Omega \rightarrow[0, \infty)$ is continuous, and $f(\cdot, \mathbf{x})$ is nondecreasing for each $\mathbf{x} \in \Omega$, and

$\left(\mathbf{A} 2^{\prime}\right) \quad g: \mathbf{R} \times \Omega \rightarrow(0, \infty)$ is continuous, and $g(\cdot, \mathbf{x})$ is nonincreasing for each $\mathbf{x} \in \Omega$.

Remark 2. If only conditions (A1)-(A3) and (L) hold, the proof for the lemma still gives $\varphi \leq u$ on $\bar{\Omega}$. A similar remark applies to the upper solution $\psi$.

Remark 3. Consider the equation

$$
f(u, \nabla u, \mathbf{x}) u_{x x}+u_{y y}+p(\mathbf{x}) g(u, \mathbf{x})=0,
$$

subject to the boundary condition (2.2). In addition to (A1), suppose that $f(u, \cdot, \mathbf{x}) \in C^{1}$ for each $u \in \mathbf{R}$ and $\mathbf{x} \in \Omega$. Then if we have strict upper and lower solutions, Lemma 1 can be generalized to include this situation. The only modification to the proof is in inequality (2.9) below. We just write

$$
(f(\hat{\psi}, \nabla \hat{\psi}, \mathbf{x})-f(u, \nabla u, \mathbf{x})) \hat{\psi}_{x x}
$$

as

$$
(f(\hat{\psi}, \nabla \hat{\psi}, \mathbf{x})-f(u, \nabla \hat{\psi}, \mathbf{x})) \hat{\psi}_{x x}+(f(u, \nabla \hat{\psi}, \mathbf{x})-f(u, \nabla u, \mathbf{x})) \hat{\psi}_{x x} .
$$

Indeed, we can have a coefficient $h(u, \nabla u, \mathbf{x})$ for $u_{y y}$ with $h$ satisfying an assumption similar to that imposed on $f$. We then have to check the additional conditions that $\psi_{y y} \leq 0$ and $\varphi_{y y} \leq 0$. 
Proof. By translation, we may assume, without loss of generality, that $y \geq 0$. For any $\varepsilon>0$, define $\hat{\psi}=\psi+\varepsilon\left(1-e^{-y}\right)$. Therefore we have $\left.\hat{\psi}\right|_{\partial \Omega} \geq\left. u\right|_{\partial \Omega}$, and

$$
\begin{aligned}
& f(\hat{\psi}, \mathbf{x}) \hat{\psi}_{x x}+\hat{\psi}_{y y}+p(\mathbf{x}) g(\hat{\psi}, \mathbf{x}) \\
&=f(\psi, \mathbf{x}) \psi_{x x}+(f(\hat{\psi}, \mathbf{x})-f(\psi, \mathbf{x})) \psi_{x x}+\psi_{y y}-\varepsilon e^{-y} \\
&+p(\mathbf{x}) g(\psi, \mathbf{x})+p(\mathbf{x})(g(\hat{\psi}, \mathbf{x})-g(\psi, \mathbf{x})) \\
& \leq f(\psi, \mathbf{x}) \psi_{x x}+\psi_{y y}+p(\mathbf{x}) g(\psi, \mathbf{x})-\varepsilon e^{-y} \\
&< f(\psi, \mathbf{x}) \psi_{x x}+\psi_{y y}+p(\mathbf{x}) g(\psi, \mathbf{x}) \\
& \leq 0 .
\end{aligned}
$$

Thus $\hat{\psi}$ satisfies inequality (2.5) with the strict inequality and inequality (2.6). We now claim that $u \leq \hat{\psi}$ on $\bar{\Omega}$. For if it is not, then there exists a $\mathbf{x}_{0} \in \Omega$ such that $\hat{\psi}\left(\mathbf{x}_{0}\right)<u\left(\mathbf{x}_{0}\right)$, and $\mathbf{x}_{0}$ is a negative local minimum of $\hat{\psi}-u$. Subtract equation (2.1) from inequality (2.8) and evaluate at $\mathbf{x}=\mathbf{x}_{0}$; we arrive at

$$
f(u, \mathbf{x})(\hat{\psi}-u)_{x x}+(\hat{\psi}-u)_{y y}+(f(\hat{\psi}, \mathbf{x})-f(u, \mathbf{x})) \hat{\psi}_{x x}<p(\mathbf{x})(g(u, \mathbf{x})-g(\hat{\psi}, \mathbf{x})),
$$

which in turn leads to

$$
f(u, \mathbf{x})(\hat{\psi}-u)_{x x}+(\hat{\psi}-\cdot u)_{y y}<0 .
$$

However this contradicts the assumption that $\mathbf{x}_{0}$ is a local minimum. Thus $u \leq \hat{\psi}$ on $\bar{\Omega}$. Taking the limit as $\varepsilon \rightarrow 0$, we have $u \leq \psi$ on $\bar{\Omega}$.

Now we take $\underline{\varphi}=\varphi$ if the strict inequality (2.3) holds. In case that $\varphi \geq \delta>0$ and strict inequality in (2.3) is replaced by inequality, we define

$$
\underline{\varphi}=\varphi-\varepsilon\left(1-e^{-y}\right) \text {. }
$$

By taking $\delta>\varepsilon>0$, we can ensure that $\underline{\varphi}>0$ on $\bar{\Omega}$. By a calculation similar to inequality $(2.8)$, we have

$$
f(\underline{\varphi}, \mathbf{x}) \underline{\varphi}_{x x}+\underline{\varphi}_{y y}+p(\mathbf{x}) g(\underline{\varphi}, \mathbf{x})>0 .
$$

In addition $\varphi$ also satisfies inequality (2.6). We claim that $u \geq \underline{\varphi}$ on $\bar{\Omega}$. For if it is not, then there exists a $\mathbf{x}_{0} \in \Omega$ such that $0<u\left(\mathbf{x}_{0}\right)<\varphi\left(\mathbf{x}_{0}\right)$, and $\mathbf{x}_{0}$ is a local positive maximum of $\underline{\varphi}-u$. Subtract equation (2.1) from inequality (2.10) and evaluate at $\mathbf{x}=\mathbf{x}_{0}$; we get

$f(u, \mathbf{x})(\underline{\varphi}-u)_{x x}+(\underline{\varphi}-u)_{y y}+(f(\underline{\varphi}, \mathbf{x})-f(u, \mathbf{x})) \underline{\varphi}_{x x}>p(\mathbf{x})(g(u, \mathbf{x})-g(\underline{\varphi}, \mathbf{x}))$,

which in turn leads to

$$
f(u, \mathbf{x})(\underline{\varphi}-u)_{x x}+(\underline{\varphi}-u)_{y y}>0 .
$$

However this contradicts the assumption that $\mathbf{x}_{0}$ is a local maximum. Thus $u \geq \underline{\varphi}$ on $\bar{\Omega}$. Taking the limit as $\varepsilon \rightarrow 0$, we have $u \geq \varphi$ on $\bar{\Omega}$. Thus the inequalities in (2.7) hold, and the proof of the lemma is complete.

\section{THE PROOF OF THEOREM 1}

In order to show the existence of a solution to the equations (1.3)-(1.4), we need to employ a corresponding result for equations (1.1)-(1.2). Such a result can be found in [4]. 
Theorem 2. Let $\Omega \subset \mathbf{R}^{N}, N \geq 1$, be a bounded domain with smooth boundary $\partial \boldsymbol{\Omega}$ (of class $C^{2+\alpha}, 0<\alpha<1$ ). If $p \in C^{\alpha}(\overline{\boldsymbol{\Omega}}), p(\mathbf{x})>0$ for all $\mathbf{x} \in \bar{\Omega}$ and $\gamma>0$, then there exists a unique function $u \in C^{2+\alpha}(\Omega) \cap C(\bar{\Omega})$ such that $u(\mathbf{x})>0$ for all $\mathbf{x} \in \Omega$ and $u$ is a solution of (1.1)-(1.2). If $\phi_{1}$ denotes an eigenfunction corresponding to the smallest eigenvalue $\lambda_{1}$ of the problem $\Delta \phi+\lambda \phi=0,\left.\phi\right|_{\partial \Omega}=0$ such that $\phi_{1}(\mathbf{x})>0$ on $\Omega$ and $\gamma>1$, then there exist positive constants $b_{1}$ and $b_{2}$ such that $b_{1} \phi_{1}(\mathbf{x})^{2 /(1+\gamma)} \leq u(\mathbf{x}) \leq b_{2} \phi_{1}(\mathbf{x})^{2 /(1+\gamma)}$ on $\bar{\Omega}$. In addition, for any $\gamma>0, u(\mathbf{x}) \geq b_{3} \phi_{1}(\mathbf{x})$ for some $b_{3}>0$.

Since $\Omega$ is bounded, we can make a translation of the domain so that it lies in the interior of the strip $\mathbf{R} \times[0, L]$ for some $L>0$. Define $\psi$ as the solution of the equations

$$
\begin{aligned}
& \psi_{y y}+\frac{M+1}{\psi^{b}}=0, \\
& \psi(0)=\psi(L)=0 .
\end{aligned}
$$

By Theorem 2, a solution $\psi$ of (3.1)-(3.2) exists in $C^{2}(0, L) \cap C[0, L]$.

Thus there exists a $\delta_{0}>0$ such that $\psi \geq \delta_{0}$ for all $\mathbf{x} \in \bar{\Omega}$.

Let $r=a-b$, which is a positive constant. Then for any $\varepsilon>0$, we define

$$
f_{\varepsilon}(u, \mathbf{x})= \begin{cases}(\psi(\mathbf{x}))^{r}, & u \geq \psi(\mathbf{x}), \\ u^{r}, & \varepsilon \leq u \leq \psi(\mathbf{x}), \\ \varepsilon^{r}, & u \leq \varepsilon,\end{cases}
$$

and

$$
g_{\varepsilon}(u, \mathbf{x})= \begin{cases}1 /(\psi(\mathbf{x}))^{b}, & u \geq \psi(\mathbf{x}), \\ 1 / u^{b}, & \varepsilon \leq u \leq \psi(\mathbf{x}), \\ 1 / \varepsilon^{b}, & u \leq \varepsilon\end{cases}
$$

It can then be checked that $f_{\varepsilon}$ and $g_{\varepsilon}$ are uniform Lipschitz continuous on $[0, \infty) \times \bar{\Omega}$. The Lipschitz constant will depend on $\varepsilon$.

Define $\Omega_{\varepsilon} \subset \subset \Omega$ such that for any $\mathbf{x} \in \partial \Omega_{\varepsilon}, \operatorname{dist}(\mathbf{x}, \partial \Omega)=\varepsilon / b_{3}$, where $b_{3}$ is as in Theorem 2. Now employing Theorem 12.5, p. 305 of [3], we ensure the existence of a solution $u_{\varepsilon} \in C^{2}\left(\Omega_{\varepsilon}\right) \cap C\left(\overline{\Omega_{\varepsilon}}\right)$ which satisfies the equations

$$
\begin{aligned}
f_{\varepsilon}\left(u_{\varepsilon}, \mathbf{x}\right)\left(u_{\varepsilon}\right)_{x x}+\left(u_{\varepsilon}\right)_{y y}+p(\mathbf{x}) g_{\varepsilon}\left(u_{\varepsilon}, \mathbf{x}\right) & =0, \\
\left.u_{\varepsilon}\right|_{\partial \Omega_{\varepsilon}} & =\varepsilon .
\end{aligned}
$$

Now for any $\mathbf{x} \in \Omega_{\varepsilon}$,

$$
\begin{aligned}
f_{\varepsilon}(\psi & , \mathbf{x}) \psi_{x x}+\psi_{y y}+p(\mathbf{x}) g_{\varepsilon}(\psi, \mathbf{x}) \\
& =0-(M+1) / \psi^{b}+p(\mathbf{x}) g_{\varepsilon}(\psi, \mathbf{x}) \\
& =-(M+1) g_{\varepsilon}(\psi, \mathbf{x})+p(\mathbf{x}) g_{\varepsilon}(\psi, \mathbf{x}) \\
& \leq-g_{\varepsilon}(\psi, \mathbf{x})<0 .
\end{aligned}
$$

In addition $\psi_{x x} \leq 0$; hence $\psi$ can serve as an upper solution.

Similarly if we define $\varphi=\varepsilon$, then

$$
f_{\varepsilon}(\underline{\varphi}, \mathbf{x}) \underline{\varphi}_{x x}+\underline{\varphi}_{y y}+p(\mathbf{x}) g_{\varepsilon}(\underline{\varphi}, \mathbf{x})>0
$$


and $\underline{\varphi}_{x x} \leq 0$. So $\underline{\varphi}$ is a strict lower solution. If $\varepsilon$ is sufficiently small, then $\psi>\varphi$ on $\overline{\Omega_{\varepsilon}}$. By using Lemma 1 (and the corresponding remarks), we can conclude that

$$
\varepsilon \leq u_{\varepsilon} \leq \psi \quad \text { on } \Omega_{\varepsilon} .
$$

Therefore we can remove the cut-off, and $u_{\varepsilon}$ satisfies

$$
\begin{aligned}
u_{\varepsilon}^{r}\left(u_{\varepsilon}\right)_{x x}+\left(u_{\varepsilon}\right)_{y y}+\frac{p(\mathbf{x})}{u_{\varepsilon}^{b}} & =0, \\
\left.u_{\varepsilon}\right|_{\partial \Omega_{\varepsilon}} & =\varepsilon .
\end{aligned}
$$

In order to extract a solution to equations (1.3)-(1.4) from the sequence $\left\{u_{\varepsilon}\right\}$, we need to show that the sequence is bounded away from zero in any compact subset of $\Omega$. Again we prove this fact by our comparison lemma.

For any $\mathbf{x} \in \Omega$, we take a circle $S$ centered at $\mathbf{x}$ with $S \subset \subset \Omega$. Without loss of generality we can regard $\mathbf{x}$ as the origin. Letting the radius of $S$ be $R$ and $\delta>0$, we then define

$$
\varphi=\delta\left(R^{2}-x^{2}-y^{2}\right)
$$

Hence $\left.\varphi\right|_{\partial S}=0, \varphi>0$ on $S$, and $\varphi_{x x} \leq 0$. Moreover for $x \in S$,

$$
\begin{aligned}
& \varphi^{r} \varphi_{x x}+\varphi_{y y}+p(\mathbf{x}) / \varphi^{b} \\
& \quad \geq-2 \delta^{1+r}\left(R^{2}-x^{2}-y^{2}\right)^{r}-2 \delta+m /\left(\delta^{b}\left(R^{2}-x^{2}-y^{2}\right)^{b}\right) \\
& \quad \geq-2 \delta^{1+r} R^{2 r}-2 \delta+m /\left(\delta^{b} R^{2 b}\right) \geq 1,
\end{aligned}
$$

by choosing $\delta=\delta(m, R, r, b)=\delta(m, \mathbf{x}, a, b)$ sufficiently small. Hence $\varphi$ is a strict lower solution. Still employing the solution $\psi$ as defined in equations (3.1)-(3.2) as our upper solution, we can apply Lemma 1 on the circle $S$ to conclude

$$
\delta\left(R^{2}-x^{2}-y^{2}\right) \leq u_{\varepsilon} \leq \psi
$$

on $\bar{S}$ for any $\varepsilon$, provided $S \subset \Omega_{\varepsilon}$.

We restrict our attention to the domain $3 S / 4$, which is the circle centered at the origin with radius $3 R / 4$. We now treat equations (3.8)-(3.9) as a linear equation, and apply Theorem 12.4, p. 302, [3] to obtain a priori estimates. Thus there exist $\gamma=\gamma(\delta, M), C=C(\delta, M)$ such that

$$
\left\|u_{\varepsilon}\right\|_{C^{1+\gamma}(\overline{S / 2})} \leq C\left(\left\|u_{\varepsilon}\right\|_{L^{\infty}(\overline{3 S / 4})}+\left\|p / u_{\varepsilon}^{b}\right\|_{L^{\infty}(\overline{3 S / 4})} \leq k,\right.
$$

where $k=k(M, m, \mathbf{x}, a, b)$ which is independent of $\varepsilon$. Using an interior Schauder estimate (Theorem 6.2, p. 90, [3]) on the circle $S / 2$, we can bootstrap to obtain

$$
\left\|u_{\varepsilon}\right\|_{\left.C^{2+\gamma} \overline{S / 4}\right)} \leq C_{1}\left(\left\|u_{\varepsilon}\right\|_{L^{\infty}(\overline{S / 2})}+\left\|p / u_{\varepsilon}^{b}\right\|_{C^{\gamma}(\overline{S / 2})}\right) \leq k_{1},
$$

where $C_{1}$ can depend on the $C^{\gamma}(\overline{S / 2})$ bound on $u_{\varepsilon}$, and $k_{1}=k_{1}(M, m, \mathbf{x}, a, b)$ for any $\varepsilon$. Hence there exists a subsequence $u_{\varepsilon} \rightarrow v$ in $C^{2}(\overline{S / 4})$. It is also clear from inequality (3.11) that $v(\mathbf{x}) \geq \delta>\mathbf{0}$, where $\delta$ can depend on $\mathbf{x}$. In other words, $v>0$ on $\Omega$. Together with the fact that $u_{\varepsilon}$ is a solution of equations (3.8)-(3.9), $v$ will satisfy 


$$
v^{r} v_{x x}+v_{y y}+\frac{p(\mathbf{x})}{v^{b}}=0
$$

on $\overline{S / 4}$. Now any compact subset $\overline{\Omega_{1}}$ of $\Omega$ can be covered with a finite number of circles $S_{i}$ such that $4 S_{i}$ still lies inside $\Omega$. The previous argument immediately gives a subsequence of $u_{\varepsilon}$ which converges to $v \in C^{2}\left(\overline{\Omega_{1}}\right)$ with $v$ satisfying equation (3.14). Let $\Omega_{1} \rightarrow \Omega$. By taking a diagonal subsequence if necessary, $v \in C^{2}(\Omega)$ will satisfy the equation (3.14) on the whole domain $\Omega$. Standard regularity estimates also give $v \in C^{2+\alpha}(\Omega)$ fron equation (3.14).

In order for $v$ to qualify as a solution of equations (1.3)-(1.4), we have to show that $v \in C(\bar{\Omega})$ and $\left.v\right|_{\partial \Omega}=0$. This we now do in the case of a convex domain.

Since $\Omega$ is convex, for any $\mathbf{x} \in \partial \Omega$, there is a tangent line $T_{\mathbf{x}}$ passing through $\mathbf{x}$ with $\Omega$ lying on one side of $T_{\mathbf{x}}$. We can also construct another line $T_{\mathbf{x}}^{\prime}$ parallel to $T_{\mathbf{x}}$ such that $\Omega$ is contained in the semi-infinite strip enclosed by $T_{\mathbf{x}}$ and $T_{\mathbf{x}}^{\prime}$. Take the orthogonal coordinate axes $x_{1}$ and $y_{1}$ so that the $x_{1}$-axis is parallel to $T_{\mathbf{x}}$. Let $\beta$ be the angle that the $x_{1}$-axis makes with the $x$-axis. It can be checked that $y_{1}=-x \sin \beta+y \cos \beta$.

We will distinguish two cases.

Case (i): $T_{\mathbf{x}}$ is not parallel to the $y$-axis. Thus $\beta \neq \pi / 2$ or $3 \pi / 2$. Throughout case (i), $\beta$ will be fixed.

Define $\bar{\phi}=\bar{\phi}\left(y_{1}\right)$ such that it solves

$$
\frac{\partial^{2} \bar{\phi}}{\partial y_{1}^{2}}+\frac{M}{\bar{\phi}^{b}}=0,
$$

subject to the boundary conditions

$$
\left.\bar{\phi}\right|_{T_{\mathbf{x}}}=0,\left.\quad \bar{\phi}\right|_{T_{\mathrm{x}}^{\prime}}=0 .
$$

Hence $\bar{\phi} \in C(\bar{\Omega}) \cap C^{2}(\Omega), \bar{\phi}>0$, and $\partial^{2} \bar{\phi} / \partial y_{1}^{2} \leq 0$ on $\Omega$.

Let $\phi(x, y)=k \bar{\phi}\left(y_{1}(x, y)\right) / \cos ^{2} \beta$ with $k \geq 1$. Simple calculations show that $\partial^{2} \phi / \partial x^{2}=k \tan ^{2} \beta \partial^{2} \bar{\phi} / \partial y_{1}^{2} \leq 0$, and $\partial^{2} \phi / \partial y^{2}=k \partial^{2} \bar{\phi} / \partial y_{1}^{2}$. The function $\phi$ is in fact an upper solution for equation (3.8), because

$$
\begin{aligned}
\phi^{r} \phi_{x x} & +\phi_{y y}+p(\mathbf{x}) / \phi^{b} \leq \phi_{y y}+p(\mathbf{x}) / \phi^{b} \\
& =k \partial^{2} \bar{\phi} / \partial y_{1}^{2}+p(\mathbf{x})(\cos \beta)^{2 b} /\left(k^{b} \bar{\phi}^{b}\right) \\
& \leq-k M / \bar{\phi}^{b}+p(\mathbf{x}) /\left(k^{b} \bar{\phi}^{b}\right) \\
& \leq 0 .
\end{aligned}
$$

Due to the growth rate of $\bar{\phi}$ as given by Theorem 2 near the boundary, by taking larger $k$ if necessary, we can always ensure that $\left.\phi\right|_{\partial \Omega_{\varepsilon}} \geq \varepsilon$. It is noted that $k$ does not depend on $\varepsilon$. Hence by Lemma 1 (and Remark 2 following it), we have $u_{\varepsilon} \leq \phi$ on $\overline{\Omega_{\varepsilon}}$. Taking the limit as $\varepsilon \rightarrow 0$, we obtain $v \leq \phi$ on $\overline{\Omega_{\varepsilon}}$. Since $\Omega_{\varepsilon}$ can be arbitrarily close to $\Omega$, it follows that

$$
0<v \leq \phi \quad \text { on } \Omega .
$$

Case (ii): $T_{\mathbf{x}}$ is parallel to the $y$-axis. 
Define $\phi=\phi(x)$ such that it solves

$$
\phi^{r} \frac{\partial^{2} \phi}{\partial y_{1}^{2}}+\frac{M}{\phi^{b}}=0,
$$

subject to the boundary conditions

$$
\left.\phi\right|_{T_{\mathbf{x}}}=0,\left.\quad \phi\right|_{T_{\mathbf{x}}^{\prime}}=0 ;
$$

hence $\phi^{r} \phi_{x x}+\phi_{y y}+p(\mathbf{x}) / \phi^{b} \leq 0$. Similarly, $\left.\phi\right|_{\Omega_{\varepsilon}} \geq \varepsilon$ and the inequalities in (3.17) hold.

Now in both cases, define $\left.v\right|_{\partial \Omega}=0$. Take any $\mathbf{x} \in \partial \Omega$, and $\mathbf{y} \in \Omega$. Since

$$
|v(\mathbf{y})-v(\mathbf{x})|=v(\mathbf{y}) \leq \phi(\mathbf{y}) \rightarrow 0
$$

as $\mathbf{y} \rightarrow \mathbf{x}, v$ is continuous at $\mathbf{x} \in \partial \Omega$. Since $\mathbf{x}$ can be any arbitrary point on $\partial \Omega, v \in C(\bar{\Omega}) \cap C^{2}(\Omega)$, and it satisfies both equation (1.3) and the boundary condition (1.4). This completes the proof of Theorem 1.

\section{SOME OPEN PROBLEMS}

We conclude this paper with a short list of some of the problems which, at the time of writing, remain open.

1. We have not established uniqueness of the solutions even though this is clear in the corresponding case where the singularity is the same in the $x$-and $y$-coordinates.

2. We have not established existence, even in two dimensions, for the nonconvex domain.

3. We have not succeeded in establishing results for more than two dimensions.

4. It seems that it would be desirable to obtain more precise estimates for the behaviour of the function and its gradient near the boundary. Perhaps the methods of [2] could give better information by flattening the boundary.

5. It seems natural to speculate that there should be results for more general types of quasilinear equations such as

$$
\begin{aligned}
F_{1}(u, \nabla u) u_{x x}+f_{2}(u, \nabla u) u_{y y}+p(\mathbf{x}) & =0, \\
\left.u\right|_{\partial \Omega} & =0,
\end{aligned}
$$

where the functions $F_{1}, F_{2}$ are monotone increasing and go to zero rapidly as $u$ goes to zero.

\section{REFERENCES}

1. S. Čanić, and B. L. Keyfitz, An elliptic problem arising from the unsteady transonic small disturbance equation, preprint.

2. M. G. Crandall, P. H. Rabinowitz, and L. Tartar, On a Dirichlet problem with a singular nonlinearity, Comm. Partial Differential Equations 2 (1977), 193-222.

3. D. Gilbarg and N. S. Trudinger, Elliptic partial differential equations of second order, 2 nd ed., Springer-Verlag, 1983.

4. A. C. Lazer and P. J. McKenna, On a singular nonlinear elliptic boundary value problem, Proc. Amer. Math. Soc. 111 (1991), 721-730.

5. A. Nachman and A. Callegari, A nonlinear singular boundary value problem in the theory of pseudoplastic fluids, SIAM J. Appl. Math. 28 (1986), 271-281. 
6. C A. Stuart, Existence theorems for a class of nonlinear integral equations, Math. Z. 137 (1974), 49-66.

7. S. D. Taliaferro, A nonlinear singular boundary value problem, Nonlinear Analysis TMA 3 (1979), 897-904.

(Y. S. Choi and A. C. Lazer) Department of Mathematics, University of Connecticut, Storrs, Connecticut 06268

E-mail address: choi@math.uconn.edu

(P. J. McKenna) Department of Mathematics and Computer Science, University of Miami, Coral Gables, Flcrida 33124

E-mail address: mckenna@math.uconn.edu 